\title{
Party Like a Russian - Zur kulturdidaktischen Analyse von Musikvideos im universitären Unterricht
}

Olga Caspers (Salzburg), Marina Scharlaj (Dresden)

Авторы статьи анализируют музыкальные видеоклипы и демонстрируют возможности их использования в преподавании культурологии и РКИ. Работа с таким комплексным явлением требует, с одной стороны, особого АиАактико-методического подхода, с Аругой стороны, Аает возможность анализа на нескольких уровнях, что обеспечивает отход от текстоцентричности традиционных материалов и способствует развитию необходимых языковых, методических и культурологических компетенций.

Иностранному студенту-русисту важно знание не только классической, но и массовой культуры, транслирующей современные поведенческие коды. В Аиалоге с неканоническими произведениями поп-культуры студенты знакомятся с повседневной культурой изучаемого языка, находят в ней черты своей собственной, учатся выявАять вариативность внутри одной культуры и заимствования из Аругих культур. Систематизация и структурный подхоА преАлагаемой модели позволяет эффективно сформировать и развить у студентов навыки социо- и межкультурной коммуникации. Исходя из этого, статья отвечает на вопросы: Насколько эффективен метод работы с музыкальными видеороликами и в чем его преимущество в культурологическом и языковом планах? При помощи каких методов и теорий возможен культурологический анализ мультимедийных материалов? Каким образом можно обеспечить трансфер современных теоретических знаний в области культурологии и АиАактики и уйти от методологически устаревших страноведения и мингводидактики?

Anka Bergmann, Olga Caspers \& Wolfgang Stadler (Hg.)

Didaktik der slawischen Sprachen - Beiträge zum 1. Arbeitskreis in Berlin (12.-14.9.2016)

(C) 2018 innsbruck university press, ISBN 978-3-903187-11-5, DOI 10.15203/3187-11-5 


\section{Einleitung}

Am 30. September 2016 brachte Robbie Williams, einer der erfolgreichsten Stars der zeitgenössischen Popkultur, die erste Single-Auskopplung Party Like A Russian heraus, die das Erscheinen seines neuen Albums The Heavy Entertainment Show ankündigte. Den Musikvideoclip (MVC) zu diesem Song, welcher auf der offiziellen Seite des Briten als Promotion zum Album veröffentlicht wurde ${ }^{1}$, haben sich innerhalb nur eines Tages über dreieinhalb Millionen Menschen angesehen. Noch am gleichen Tag erschien dieses Musikvideo auf YouTube mit russischen Untertiteln ${ }^{2}$ und wurde aktiv auf russischsprachigen Internetforen sowie in sozialen Netzwerken besprochen. Auch der staatliche TV-Sender Rossija 24 sendete an diesem Tag eine Reportage über das Musikvideo von Robbie Williams, in der die ModeratorInnen die Meinung äußerten, das Video ${ }^{3}$ präsentiere das aktuelle, klischeehafte Bild von Russland im Westen und diene somit dazu, die schwindende Beliebtheit des Sängers zu retten.

Was hier als eine Marketingstrategie herausgestellt wurde, kann ebenso als eine ironische Antwort auf überzogene Inszenierungspraktiken im heutigen Russland verstanden werden. Der Videoclip von Williams thematisiert das exzessive Leben eines Oligarchen und bedient sich einer Ästhetik des Glamours, die in der westlichen Popkultur verankert und gleichzeitig signifikant für die Formierung einer neuen nationalen Idee in Russland ist (vgl. dazu Goscilo \& Strukov 2010, Menzel 2013, Zvereva 2008). Verkörperte vor ca. zehn Jahren die ostentative Selbstdarstellung, die u. a. in Vladimir Putins öffentlichen Auftritten zu beobachten war, die Ideologie des wirtschaftlichen Erfolges des Landes, so wird der ästhetische Modus des Glamours heute insb. für die Vermittlung politischer Inhalte und die Inszenierung des Hurra-Patriotismus genutzt. Die Ausbildung einer patriotischen Weltanschauung findet dabei zunehmend in populärkulturellen Formaten statt, die - unter Rekurs auf, hollywoodeske 'Muster - das positive und heroische Selbstbild von Russland transportieren.

1 http://tinyurl.com/aa4yq [02.12.2017].

2 http://tinyurl.com/z2fy6ls [02.12.2017].

3 Vesti vom 30.09.2016: https://tinyurl.com/y9o3cz2b [02.12.2017].

Anka Bergmann, Olga Caspers \& Wolfgang Stadler (Hg.)

Didaktik der slawischen Sprachen - Beiträge zum 1. Arbeitskreis in Berlin (12.-14.9.2016)

(C) 2018 innsbruck university press, ISBN 978-3-903187-11-5, DOI 10.15203/3187-11-5 
Ein inhaltliches und ästhetisches Pendant zum Videoclip von Robbie Williams bildet vor diesem Hintergrund das 2015 bekannt gewordene russische Lied von Oleg Gazmanov Vpered, Rossija $a^{4}$ Wie in einem Imagefilm werden im Videoclip von Gazmanov die Schauplätze der Erfolge Russlands fokussiert - in einer genauso stereotypen wie glamourösen Art und Weise. Beide Videoclips, die einem transkulturellen Genre der Popmusik angehören, zeigen exemplarisch die unterschiedlichen (vom nationalen Kontext abhängigen) Funktionsweisen dieser auf. Sie liefern eine Vielzahl von Auto- und Heterostereotypen und sind Teil einer Bild- und Medienkultur, die sowohl globale als auch kulturspezifische Merkmale aufweist.

Bezeichnend ist, dass auch die russische Musikszene auf überladene mediale, von der militärischen Symbolik dominierte, patriotische wie populistische Inszenierungen wie die von Gazmanov kritisch reagiert. Als eine wichtige Plattform für Kritik erweist sich jedoch eine andere Art der Musik, etwa Rock oder Skacore. So persifliert der MVC Patriot $(2016)^{5}$ der Band Nesčastnyj slučaj die wachsende Bedeutung des Hurra-Patriotismus, indem er einen ,wahren' Patrioten wie den Regisseur Nikita Michalkov satirisch nachzeichnet und seine typischen Eigenschaften in einer stilistischen Mischung von Popmusik und Folklore verspottet. Die populäre exzentrische Band Leningrad behandelt das GlamourThema als ein Massenphänomen, das bereits die untere Mittelschicht erfasste (s. dazu Gussarowa 2008: 4). Im Gegensatz zu den oben genannten Videos, in denen männliche Protagonisten die Hauptrolle spielen und alle Attribute eines glamourösen Lebens aufweisen, handelt es sich im Lied Eksponat $(2016)^{6} \mathrm{um}$ eine junge Frau aus bescheidenen Verhältnissen, die versucht, das äußere Erscheinungsbild der Elite zu imitieren. Die Protagonistin täuscht die Glamour-

4 Auf dem YouTube-Kanal des Sängers wurde dieser Videoclip über fünf Millionen Mal aufgerufen, s. https:// tinyurl.com/lwq65vl [02.12.2017].

5 https://tinyurl.com/h54ompq [02.12.2017].

6 https://tinyurl.com/jz7me6f [02.12.2017]. Das Video erreichte nur innerhalb der ersten zwei Wochen nach seinem Erscheinen auf YouTube ca. 70 Millionen Klicks. Inzwischen gilt der als Video des Jahres ausgezeichnete MVC mit über 114.985.406 Aufrufen als erfolgreichster Song der Band. Nachdem der Ausdruck „Na Labutenach" aus dem Song mehrfach in Social Media geteilt wurde, wurde er als viraler Post zum Meme. Nach Angaben der russischen Suchmaschine Yandex belegte dieses Meme den ersten Platz unter den bekanntesten Internet-Memen des Jahres 2016 (https://tinyurl.com/y7qhlavv [02.12.2017]).

Anka Bergmann, Olga Caspers \& Wolfgang Stadler (Hg.)

Didaktik der slawischen Sprachen - Beiträge zum 1. Arbeitskreis in Berlin (12.-14.9.2016)

(C) 2018 innsbruck university press, ISBN 978-3-903187-11-5, DOI 10.15203/3187-11-5 
Attribute vor, um in die Welt der Schönen und Reichen zu gelangen und dort den erwünschten sozialen Aufstieg durch Heirat zu erzielen. Karikiert wird hier der Alltag einer durchschnittlichen russischen Familie, die sich am täglichen Medienkonsum beteiligt.

Diese Beispiele machen deutlich, dass die populären Musikvideos nicht nur der Unterhaltung dienen, sondern latent Deutungsmuster für die gesellschaftliche Wirklichkeit transportieren. Somit verfügen die Videos über ein hohes didaktisches Potenzial, welches im Unterricht sowohl sprachlich als auch inter- und soziokulturell genutzt werden kann. Basierend auf dieser Prämisse fragen wir im vorliegenden Beitrag insb. nach dem kulturdidaktischen Mehrwert der Arbeit mit MVCs in der universitären Ausbildung von RussistInnen. An ausgewählten Beispielen wollen wir zum einen das interkulturelle Lernpotenzial von Videoclips eruieren sowie didaktisch-methodische Verfahren zur Entwicklung interkultureller Kompetenz der RussischlernerInnen skizzieren. Zum anderen geht es uns im Folgenden um eine kulturwissenschaftliche Rahmung des Materials und damit um Modelle, die in der theoriegeleiteten Lehre zur Vermittlung soziokultureller und interkultureller Inhalte eingesetzt werden können. In dieser disziplinären Perspektive versteht sich unser Beitrag als Anregung zur Diskussion über die Transferleistung zwischen kulturwissenschaftlichen und fachdidaktischen Inhalten, die zwar von FremdsprachendidaktikerInnen bereits eingefordert wurde (vgl u. a. Hallet \& Nünning 2007), im Fach Russisch bisher jedoch wenig Beachtung erfahren hat.

Im Vergleich zu anderen Fachbereichen wie der Linguistik und Literaturwissenschaft spielt die Kulturwissenschaft offensichtlich immer noch eine geringere Rolle in der Didaktik der slawischen Sprachen. ${ }^{7}$ Hinzu kommt die Tatsache, dass sowohl im kulturwissenschaftlichen als auch im landeskundlichen universitären Russischunterricht der Gegenstandsbezug traditionell auf die sog. Hochkultur eingegrenzt wird. Interkulturelles Lernen erfolgt im fachwissenschaftlichen Teil der RussischlehrerInnenausbildung häufig auf der Grundlage von literarischen

7 Diese Aussage bezieht sich auf Studienordnungen und Lehrverzeichnisse deutschsprachiger Universitäten, die von uns geprüft wurden. Es fällt dabei auf, dass kulturwissenschaftliches Wissen oft in Korrespondenz mit benachbarten Disziplinen vermittelt wird. Wesentliche Impulse für die Kulturwissenschaft kommen aus der Literaturwissenschaft. Zu nennen ist ferner kulturwissenschaftliche Linguistik (v. a. Kuße 2012), die innerhalb der slawistischen Sprachwissenschaft erst seit einigen Jahren an Bedeutung gewinnt.

Anka Bergmann, Olga Caspers \& Wolfgang Stadler (Hg.)

Didaktik der slawischen Sprachen - Beiträge zum 1. Arbeitskreis in Berlin (12.-14.9.2016)

(C) 2018 innsbruck university press, ISBN 978-3-903187-11-5, DOI 10.15203/3187-11-5 
Texten (vgl. Mehlhorn 2014: 223-225). Ohne die Zweckmäßigkeit dieser Verfahren grundsätzlich in Frage zu stellen, gehen wir von der Annahme aus, dass „die Akkumulation von symbolischem Kapital“ (Schmid 2016: 292) heute nicht mehr hauptsächlich über die Lektüre des literarischen Kanons, sondern über multimediale Produkte der Kulturindustrie geschieht (vgl. ebd. sowie für Fachdidaktik z. B. Volkmann 2007). In Erzeugnisse der Populärkultur sind bestimmte ideologische Wertsysteme eingeschrieben, die von RezipientInnen aufgenommen werden und die ihr Weltbild prägen. Die breite Rezeption der eingangs genannten Videoclips macht beispielhaft deutlich, welch wichtigen Platz die Populärkultur in der modernen Mediengesellschaft sowie im Alltag der jüngeren Lernergeneration einnimmt, und u. a. aus diesem Grund im Unterricht Berücksichtigung finden soll.

Konkret wollen wir in diesem Zusammenhang prüfen, in welcher Form populäre Musik als Bestandteil der Alltagskultur der LernerInnen und als soziokulturelle Erscheinung im Unterrichtsraum behandelt werden kann. Wie lässt sich das Potenzial der Videoclips kulturdidaktisch nutzen? Welche Theorien und Methoden eignen sich für die wissenschaftsbasierte Analyse der Musikvideos, von der aus Prozesse der Sensibilisierung für das Eigene und das ,Fremdspezifische in Gang gesetzt werden? Und schließlich: Wie kann man die Rezeption der MVCs durch Strukturierung steuern und damit die Medienkompetenz der LernerInnen stärken?

\section{2. (Pop-)Musik als Gegenstand der Lehre und Forschung}

$\mathrm{Zu}$ den konventionellen Medien im schulischen und universitären Russischunterricht zählen neben den Lehrwerken auch Musik und Film. Russische Lieder wurden dabei lange Zeit im Audioformat rezipiert. Die - oft in Form von Frontalunterricht - von LernerInnen geforderten Aktivitäten umfassten das Vorlesen des Textes, das Ausfüllen von Lücken, die Wiederherstellung der Reihenfolge von Text-Fragmenten, Ausspracheübungen und schließlich die Beantwortung von Verständnis- und Interpretationsfragen zum Text. Dabei wurden Lieder zur Schulung des Hör- und Leseverstehens, zur Motivation und Auflockerung oder zur Erweiterung von soziokulturellen Kenntnissen eingesetzt. Filme dagegen sah

Anka Bergmann, Olga Caspers \& Wolfgang Stadler (Hg.)

Didaktik der slawischen Sprachen - Beiträge zum 1. Arbeitskreis in Berlin (12.-14.9.2016)

(C) 2018 innsbruck university press, ISBN 978-3-903187-11-5, DOI 10.15203/3187-11-5 
man im Unterricht gelegentlich als puren Unterhaltungszweck ohne didaktische Zielsetzung an. Mit der Entwicklung des interkulturellen Ansatzes im Fremdsprachenunterricht wurden sowohl Lieder als auch Filme als Gegenstand des interkulturellen Lernens immer wieder in den Blick genommen (Caspers 2016). Entsprechend umfangreich sind Vorschläge zur Unterrichtsgestaltung, aber auch aufbereitete Materialien für die Praxis im Bereich der Russistik (konkrete Beispiele werden regelmäßig in den Ausgaben der Zeitschrift Praxis Fremdsprachenunterricht präsentiert). Auffallend ist dabei jedoch, dass audiovisuelle Potenziale der Medien nach wie vor nicht im Vordergrund stehen. Videoclips, die sowohl musikalische als auch filmische Elemente enthalten, werden in praktischen Handreichungen sowie bei einer theoriegeleiteten didaktisch-methodischen Aufbereitung im Fach Russisch kaum beachtet.

Wesentliche Vorarbeiten zum Einsatz von MVCs im Fremdsprachenunterricht kommen aus der Anglistik und Romanistik (Blell 2002, Frazer 2005, Reimann 2014, Thaler 1999, Volkmann 2007), wobei die von Thaler erarbeitete Typologie der unterschiedlichen Formen der Präsentation von MVCs immer noch zu den wichtigsten Grundlagen in der Mediendidaktik zählt. Reimann (2014) ergänzt die Typologie von Thaler und kommentiert ihre Praktikabilität aus heutiger Sicht. Indem er die Arbeit mit Musikvideos in die Nähe der Filmdidaktik rückt, spricht er sich für den vision-first-approach aus. Der Schwerpunkt wird hier auf die Auseinandersetzung mit Bildern, einzelnen Videosequenzen sowie auf das Sehverstehen gelegt.

Grundsätzlich werden in der Fachliteratur zur Fremdsprachendidaktik immer wieder folgende Argumente für den Einsatz audiovisueller Formate angeführt: Popularität und Motivation, Bezug zur Lebenswelt der LernerInnen, Schulung rezeptiver und produktiver Fertigkeiten, Stärkung kommunikativer und interkultureller Kompetenzen, Methodenkompetenz und nicht zuletzt Förderung audiovisueller und medialer Kompetenz (vgl. Begriffe wie „audio literacy“, „visual literacy“, ,audio-visual literacy“, „media literacy“). ${ }^{8}$ Im Bereich des interkulturellen

8 An dieser Stelle seien die „A“-Charakteristika des Medienansatzes von Thaler (2012: 166) aufgelistet, mit denen sich der Einsatz von Musikclips begründen lässt: Affektivität, Anschaulichkeit, Authentizität, Abwechslung, Aktivität, Aktualität, Alltagsbezug, Adressatenorientierung.

Anka Bergmann, Olga Caspers \& Wolfgang Stadler (Hg.)

Didaktik der slawischen Sprachen - Beiträge zum 1. Arbeitskreis in Berlin (12.-14.9.2016)

(C) 2018 innsbruck university press, ISBN 978-3-903187-11-5, DOI 10.15203/3187-11-5 
Lernens handelt es sich in erster Linie um den Ausbau einer critical cultural awareness (Thaler 2012: 243). Eisenmann (2010: 253-254) bringt die Besonderheiten von MVCs auf den Punkt, die sie für den Bereich des kommunikativen und interkulturellen Lernens unverzichtbar machen: Authentizität und kultureller Bezug, kulturelle Konnotation, Zugang zu Emotionen, Komplementarität von Musik, Text und Bild. Als anschlussfähig für unser Vorhaben erweist sich der exemplarische Bezug zum populären MVC Zombie von der Gruppe The Cranberries, welchen die Autorin im Kontext des interkulturellen Lernens herstellt.

Einen weiteren Anknüpfungspunkt bildet der Sammelband Film im Fremdsprachenunterricht. Literarische Stoffe, interkulturelle Ziele, mediale Wirkung (Leitzke-Ungerer 2009), der über das klassische Spielfilmformat hinausgehend eine Reihe von Vorschlägen dazu enthält, wie man mit Pop, Rap und Musikvideos aus YouTube arbeiten kann. Hier wird der interkulturelle und kommunikative Zugang bevorzugt, wobei die theoretischen Forschungsansätze in praktischen Beispielen eine Anwendung finden. So skizziert Willwer (2009) in seinem Beitrag die wachsende Rolle der Musikvideos im Alltag der LernerInnen und weist darüber hinaus auf das veränderte Rezeptionsverhalten der jungen Generation hin, bei der das Visuelle deutlich an Relevanz gewinnt. Bei Willwer findet sich eine kulturtheoretisch basierte Charakteristik von MVCs, die Möglichkeiten einer Schnittstellenbildung zwischen Kulturwissenschaft und Fachdidaktik aufzeigt (etwa das semiotische Triasverhalten Bild-Text-Musik, Relationen zwischen SenderIn und EmpfängerIn, Typologien von Musikvideos etc.).

Volkmann (2007) favorisiert ebenfalls den semiotischen Zugang zu Produkten der Populärkultur und versteht vor diesem Hintergrund den MVC als semiotischen Text. Das Medienprodukt als „Senderinstanz“ von „Appellstrukturen“ enthält wichtige kulturelle Informationen, die es auf der Seite der RezipientInnen zu dekodieren und zu interpretieren gilt (ebd.: 282). In der Übertragung auf den Unterrichtskontext heißt das konkret, den LernerInnen Strategien des Verstehens, des Dekodierens medialer Texte zu vermitteln. Das entspricht dem rezeptionsorientierten Ansatz der Mediendidaktik (Blell 2005). In der universitären Lehre bedeutet dieses Vorgehen einerseits die Vermittlung von methodischen Kenntnissen und Fähigkeiten, andererseits die Entwicklung von theoriegeleiteten Bezugsrahmen, mit deren Hilfe eine fundierte systematische Untersuchung kul-

Anka Bergmann, Olga Caspers \& Wolfgang Stadler (Hg.)

Didaktik der slawischen Sprachen - Beiträge zum 1. Arbeitskreis in Berlin (12.-14.9.2016)

(C) 2018 innsbruck university press, ISBN 978-3-903187-11-5, DOI 10.15203/3187-11-5 
tureller Phänomene erst ermöglicht wird. Wie dies im Einzelnen im Fach Russisch theoretisch präzisiert und praktisch realisiert werden kann, soll im Folgenden diskutiert werden.

\section{Kulturwissenschaftliche Zugänge zu MVCs}

Während der Einsatz von Musikvideos im sprachpraktischen Teil der universitären Ausbildung durchaus stattfindet und außerdem auch kulturtheoretisch bereits gut erschlossen ist (insb. in den anglosächsischen cultural studies), ist die Behandlung der Popmusik in den Fachwissenschaften eher ein Randthema der Russistik. Die Erforschung der Populärkultur in den akademischen Kreisen der Slawistik stellt zwar kein Novum mehr dar, stößt jedoch ungeachtet aller Impulse aus den Nachbardisziplinen auf ein vergleichsweise geringes Interesse. ${ }^{9}$ Festzustellen ist ferner, dass sich die nach dem Ende des Ost-West-Konfliktes unternommene Übertragung der westlichen, in der Anglistik und Amerikanistik fest etablierten Beschreibungsmodelle von Alltagskultur auf den russischen Kontext als problematisch erweist. Bereits frühere Studien zur Massen- bzw. Populärkultur zeigten auf, dass die wirtschaftlichen und gesellschaftlichen Transformationen im neuen Russland nicht zur Ablösung sowjetischer Erinnerungsmuster führten (vgl. Barker 1999, Trepper 2002). Im Gegenteil: Die Glorifizierung der Sowjetunion fand sehr schnell Einzug in neue populäre Gattungen. Diese Diskrepanz auf der Gegenstands- und Analyseebene stellt sich aus unserer Sicht gerade für das interkulturelle und Medienkompetenz fördernde Lernen als Vorteil heraus. In der Fachdidaktik lassen sich die Linien der philologisch ausgerichteten Kulturwissenschaft und empirisch-soziologisch basierten cultural studies zusammenführen, indem sowohl die Hochkultur behandelt wird als

9 Dem Wechselverhältnis zwischen ost- und westeuropäischer Populärkultur ist die Nummer 5 der Zeitschrift Osteuropa (Der Osten im Westen. Importe der Populärkultur) gewidmet (Themenheft Osteuropa 2007). Ein aktueller Forschungsüberblick zur Populärkultur im russischsprachigen Raum findet sich in Rozenchol'm \& Savkina (2015). Beispiele sowie weiterführende Literatur zur „staatlichen Kulturindustrie“ in Russland sind in der (wenngleich an manchen Stellen kritisch zu hinterfragenden) Publikation von Schmid (2016) enthalten. Einige Aspekte slawischer Popkultur sind in Doschek \& Simonek (2015) beschrieben.

Anka Bergmann, Olga Caspers \& Wolfgang Stadler (Hg.)

Didaktik der slawischen Sprachen - Beiträge zum 1. Arbeitskreis in Berlin (12.-14.9.2016)

(C) 2018 innsbruck university press, ISBN 978-3-903187-11-5, DOI 10.15203/3187-11-5 
auch über die Popkultur der Zugriff auf alltagskulturelle Phänomene erfolgen kann.

Als transkulturelle Erscheinungen können die eingangs genannten Musikvideos kontrastiv mit semiotischen Instrumentarien - sowohl als versprachlichte als auch als visuelle Symbolisierungsformen - beschrieben werden. Mithilfe von Videoclips als Teil eines Text- und Diskursraums lassen sich (1) die materiale Dimension, (2) die soziale Dimension und (3) die mentale Dimension der Kultur im Sinne Posners (1991) aufzeigen. In der Terminologie von Bachmann-Medick (1996: 9f.) wird dadurch der Zugang zu „Selbstbeschreibungsdimensionen“ einer Gesellschaft gewonnen. Einen wichtigen Bezugsrahmen für die Modellierung dieser Dimensionen liefert eine konzeptorientierte Kulturdidaktik. In Blick genommen werden dabei Konzepte wie kollektive Erinnerung und kulturelles Gedächtnis, Identitätskonstruktionen, Kollektivsymbole, Zentrum und Peripherie, Eigenes und Fremdes u. ä., die modellhaft auf die Mechanismen kultureller Prozesse verweisen und exemplarisches Lernen sowie das Erwerben transferfähiger analytischer Kompetenzen ermöglichen (vgl. Sommer 2007: 193). Das In-Beziehung-Setzen der eigenen kulturellen Erfahrungen mit denen der zielsprachlichen Realität schafft eine wichtige Grundlage für interkulturell reflektiertes Verhalten. Dieser Zugang verdeutlicht beispielhaft, wie die thematischen Zugänge des soziokulturellen Ansatzes produktiv ergänzt bzw. ersetzt werden.

Ferner und für unsere Zwecke zentral: Bei der Analyse der Musikvideos gilt es, die inhaltliche Komponente um den audiovisuellen Aspekt zu erweitern. Hier greift am ehesten die Untersuchungsperspektive der Transkulturalität, in der die wachsende Heterogenität kultureller Sphären akzentuiert und dichotome, z. T. simplifizierende Muster zugunsten gemeinsamer Merkmale unterschiedlicher Kulturen aufgegeben werden (vgl. Welsch 2010). Von hier aus kann der Blick auf das Unvertraute und Unverstandene im Vertrauten gelenkt werden. Im Bereich der Popmusik stellt der visuelle, ästhetische Modus der Inszenierungen ein solches gemeinsames Merkmal dar, das den LernerInnen vertraut ist. Betrachtet man Popmusikvideos als kulturübergreifende Texte, so kann zu ihrer Analyse die Ideologie des Glamours herangezogen werden. Hier liefert die US-amerikanische Unterhaltungskultur eine Vorbildfunktion und damit einhergehend auch die Grundlage zur vergleichenden Beschreibung der popkulturellen Ästhetik.

Anka Bergmann, Olga Caspers \& Wolfgang Stadler (Hg.)

Didaktik der slawischen Sprachen - Beiträge zum 1. Arbeitskreis in Berlin (12.-14.9.2016)

(C) 2018 innsbruck university press, ISBN 978-3-903187-11-5, DOI 10.15203/3187-11-5 
In Anlehnung an westliche Konzepte assoziiert man Glamour im russischsprachigen Diskurs mit Konsumkultur, mit bestimmten kulturellen Praktiken wie Mode, Show, Lebensstil oder auch mit Medienindustrie, die durch Hochglanzmagazine, Bücher und TV die Vorbilder eines schönen hedonistischen Lebens für ein Massenpublikum liefert. Im Russland der 2000er Jahre wird außerdem die Politik als zur Glamour-Welt gehörig inszeniert und als eine neue nationale Idee interpretiert (vgl. Schmid 2016: 320-327, Zvereva 2008: 128-129). An thematisch und ästhetisch vergleichbaren Videos wie denen von Robbie Williams und Oleg Gazmanov sowie an deren satirischer Verkehrung zeigt sich exemplarisch, wie unterschiedlich globale Phänomene lokal ausgeprägt sein können. Zur Sensibilisierung der LernerInnen für diese Differenzen ist die theoriegeleitete soziokulturelle Einbettung der zu behandelnden Beispiele unabdingbar. Ansätze dazu finden sich in der kultursoziologisch orientierten Forschung (vgl. v. a. Dubin 2010).

Der Argumentation der KultursoziologInnen folgend erwies sich Glamour in der Zeit, in der Russland aus den politischen und wirtschaftlichen Problemen der 1990er Jahre herausgeführt wurde, als ein geeignetes Instrument zur Darstellung des neuen, deutlich besseren Alltags. Zugleich verhinderte die Ideologie des materiellen Erfolges und der Unterhaltung die Einmischung der BürgerInnen in die Politik (vgl. Rudova 2008: 2). Heute, in Zeiten der wirtschaftlichen Schwäche Russlands, verliert Glamour als Teil der nationalen Idee nicht an Bedeutung, sondern verschiebt den Schwerpunkt auf den politischen Erfolg (vgl. Kanzler \& Scharlaj 2017). Popkulturelle Genres werden dabei für staatliche Zwecke, zur Vermittlung des ,blendenden' heroischen Selbstbildes instrumentalisiert. Die martialischen medialen Inszenierungen, die die heutige Fernsehlandschaft, und darunter die patriotischen MVCs Russlands, dominieren, verkörpern die wiedergewonnene Stärke Russlands. Sie bieten nicht nur einen faktischen, sondern v. a. einen emotionalen Rahmen, der die Mitwirkung der ZuschauerInnen einfordert. Erzielt wird dadurch die Identifikation mit dem eigenen Land, das im TV stets in positivem Licht gezeigt wird. Die prunkvolle Formensprache sowie die Reduzierung der Aussage auf die Form und die Fragmentierung des Bildmaterials bewirken nach Dubin $(2006,2010)$ die Totalisierung des ästhetischen kulturellen Sinns, wie man diese einerseits in der eintönigen Sowjetkultur, anderseits in den schillernden Praktiken des Glamours beobachten kann. Mit anderen Worten: Im

Anka Bergmann, Olga Caspers \& Wolfgang Stadler (Hg.)

Didaktik der slawischen Sprachen - Beiträge zum 1. Arbeitskreis in Berlin (12.-14.9.2016)

(C) 2018 innsbruck university press, ISBN 978-3-903187-11-5, DOI 10.15203/3187-11-5 
Genre der patriotischen Popmusik verschränkt sich die Sowjetnostalgie, die im Inhalt dominiert, mit einer glamourösen Erscheinungsform und Ästhetik.

Vor diesem Hintergrund ist eine Sonderposition der russischen, im Gegensatz zur westlichen Populärkultur zu berücksichtigen (Scharlaj 2017). Anders als die VertreterInnen der cultural studies sieht Dubin (2010) die Spezifika der Populärkultur (als Synonym für Massenkultur) nicht in der Beschaffenheit, der Bedeutungsproduktion, in Rezeptionspraktiken, Qualität oder Struktur dieser Texte, sondern in der Kommunikation mit den AdressatInnen. Zu den kommunikativen Grundlagen der Populärkultur zählen die Bezugsherstellung zu einem virtuellen everyman sowie eine wiedererkennbare Botschaft (vgl. ebd.). Der Sinn der immer gleichbleibenden, tautologischen Kommunikation besteht dabei in der Erschaffung eines Konsensgefühls, welches die Konstituierung der Gesellschaft als einheitliche „virtuelle Masse“ oder „Konsumentengesellschaft“ begünstigt und jede Dialogizität tilgt (ebd.: 77). In dieser Interpretation ist der ästhetische Modus des Glamours geradezu prädestiniert, Kritik auszuschließen. Die westliche Populärkultur, sofern sie nicht als „mediale Hölle“ (s. dazu Thaler 1999: 249; Volkmann 2007: 279-283) verstanden wird, erfüllt dagegen eine wichtige Ventilfunktion und transportiert alternative Deutungsmuster. Sie wird schließlich als ein Ort gesehen, in dem kulturelle Bedeutungen ausgehandelt werden (mehr dazu s. Scharlaj 2017). In einem immer noch stark in der sowjetischen Tradition verankerten Russland stellt das Populäre, wie man es im Westen versteht, teilweise eine eskapistische Gegenkultur dar.

Schon diese kursorische Auswahl von Merkmalen der Glamourkultur spricht für deren Thematisierung im universitären kulturvermittelnden Unterricht. Mit Bezug auf unterschiedliche Funktionsweisen der Populärkultur können Mechanismen kultureller Prozesse zugänglich gemacht werden. In diesem Rahmen bietet es sich an, sowohl die Funktionen als auch die im Genre der Popmusik impliziten Vorstellungen über Werte, gesellschaftliche Beziehungen, kollektive Erinnerungen oder auch Bildkulturen als solche im Unterricht zu exemplifizieren. Der Hurra-Patriotismus als aktuelles Phänomen ist für die Behandlung dieser Problematik sehr gut geeignet.

Auf der Objektebene fördert der von uns vorgeschlagene Ansatz - neben der Entwicklung interkultureller Kompetenz - einen reflektierten Umgang mit

Anka Bergmann, Olga Caspers \& Wolfgang Stadler (Hg.)

Didaktik der slawischen Sprachen - Beiträge zum 1. Arbeitskreis in Berlin (12.-14.9.2016)

(C) 2018 innsbruck university press, ISBN 978-3-903187-11-5, DOI 10.15203/3187-11-5 
Medien und stärkt auf der metakognitiven Ebene einen kritischen Umgang mit kulturwissenschaftlichen Theorien. Die Vermittlung von transferierbaren methodischen Fähigkeiten und deren Überprüfung an anderen Beispielen begünstigen nicht zuletzt autonomes Lernen.

\section{Didaktisch-methodische Überlegungen und unterrichts- praktische Beispiele}

Wie oben beschrieben, gibt es in der Fremdsprachendidaktik bereits zahlreiche Publikationen zur Methodik des Einsatzes von MVCs. Im Folgenden wollen wir diese Vorschläge bezogen auf russische Beispiele adaptieren, um Impulse zur Arbeit mit diesem Medium zu liefern. Ausgehend von der These, dass MVCs ein bedeutendes Medium kultureller Repräsentation, Inszenierung und Selbstverständigung darstellen, ist unsere Analyseperspektive besonders auf die Frage gerichtet, „wie Bilder, Mythen, kollektive Erinnerungen und andere Zeugnisse des kulturellen bzw. nationalen Selbstverständnisses von Individuen aus einer Eigen- bzw. Fremdperspektive inszeniert bzw. konstruiert werden“ (Hu 2007: 17). Das übergeordnete Lernziel ist dabei, die medialen Produkte ,lesen`zu lernen, d. h. eine multimodale ästhetische Symbolisierungsform zu verstehen. Es beinhaltet die Fähigkeit zur Clipanalyse in ihren formalen, funktionalen und evaluativen Komponenten.

Um diese Fähigkeit zu entwickeln, d. h. die Musikvideoanalyse aus kulturdidaktischer Perspektive effizient und nachhaltig durchzuführen, bedarf es eines Musikvideokorpus bzw. einer praktischen Plattform, die eine Ansammlung von Beispielen enthält. Der Vorteil des MVC-Korpus besteht darin, dass daraus je nach Zielsetzung eine Kombination von Videos ausgewählt werden kann und so die Behandlung verschiedener Aspekte der Populärkultur in einer Unterrichtsreihe ermöglicht wird. Für die Zusammenstellung des Korpus sind mehrere Kriterien von Bedeutung - darunter insb. Aktualität und Relevanz für die russländische Gesellschaft und Kultur. Die MVCs sollen dabei nicht nur eine geeignete Grundlage für die Analyse zentraler gesellschaftlicher, sondern auch alltagstypischer und ästhetischer Phänomene bilden. Es empfiehlt sich ferner, mit Videos zu arbeiten,

Anka Bergmann, Olga Caspers \& Wolfgang Stadler (Hg.)

Didaktik der slawischen Sprachen - Beiträge zum 1. Arbeitskreis in Berlin (12.-14.9.2016)

(C) 2018 innsbruck university press, ISBN 978-3-903187-11-5, DOI 10.15203/3187-11-5 
die der Lebenswelt der Studierenden nahe sind und soziokulturelle Phänomene in den Mittelpunkt rücken, die sowohl affirmativ gesehen als auch kritisch hinterfragt werden können (s. auch den Beitrag von Kaltseis in diesem Band).

Die bisherigen Ausführungen zum kulturdidaktischen Potenzial der MVCs lassen sich an folgenden möglichen Korpusbeispielen konkretisieren: Die „Selbstbeschreibungsdimensionen“ der offiziellen russischen Kultur sind in die Lieder der patriotischen Estrada eingeschrieben. Zu den bekanntesten und vom Staat vielfach ausgezeichneten Sängern zählen Oleg Gazmanov, Denis Majdanov, Aleksandr Rozenbaum, Maršal u. a. Ihr Repertoire enthält eine Reihe von Liedern, die in nostalgischer bis nationalistischer Manier die (heroische) russischsowjetische und zaristische Vergangenheit revitalisieren. Die MVCs dieser Sänger sind für unsere Zwecke insofern gut geeignet, als sie das imperiale Selbstverständnis des neuen Russlands und somit auch die Wertinstanziierung,von oben ‘ deutlich machen - vgl. z. B. Lieder von Majdanov Flag moego gosudarstva (2013), Kto takie russkie (2015); Gazmanov Vpered, Rossija! (2015), Nikto, krome nas! (2015) u. v. m.

Für die Kontrastierung des positiven nationalen Selbstverständnisses bieten sich MVCs an, die alternative Lesarten zu Konzeptionen des Glamours und Patriotismus transportieren, wie etwa der Videoclip Patriot der Band Nesčastnyj slučaj (2016). Die Persiflage der glamourösen und konsumorientierten Wertaneignungen in der Alltagskultur sind zum Markenzeichen der Band Leningrad geworden: Sumka (2013), Plaču (2013), Plat'e Sen Loran (2014), Patriotka (2014), VIP (2015), Ekksponat (2016), Sis'ki (2016), Očki Sobčak (2016). Weisen die letztgenannten russischen Beispiele Merkmale des peripher Kulturellen - im Sinne Lotmans (1990) - auf, so handelt es sich im Videoclip von Robbie Williams Party Like a Russian (2016) um eine Fremdperspektive, deren Berücksichtigung das interkulturelle Lernen forciert.

Wie bereits dargelegt, erweist sich Popmusik als eine komplexe Erscheinung. Sie beinhaltet ein vielschichtiges musikalisches und kulturelles System, dessen Verständnis mehrere Kompetenzen verlangt und dessen Dekodierung eigene Strategien sowie Kenntnisse mehrerer Teildiskurse einer Kultur voraussetzt. Da die MVCs als Beziehungsgeflecht von musikalischen, performativen und medialen Faktoren fungieren, wird der/die EmpfängerIn nicht nur durch den Text

Anka Bergmann, Olga Caspers \& Wolfgang Stadler (Hg.)

Didaktik der slawischen Sprachen - Beiträge zum 1. Arbeitskreis in Berlin (12.-14.9.2016)

(C) 2018 innsbruck university press, ISBN 978-3-903187-11-5, DOI 10.15203/3187-11-5 
und die Musik, sondern auch durch deren Zusammenspiel mit der jeweiligen Bildfolge konfrontiert. Um dieser Komplexität auf der Gegenstandsebene sowie der geforderten Multiperspektivität auf der Analyseebene gerecht zu werden, ist ein systematisches Vorgehen erforderlich.

\subsection{Dimensionen der MVC-Analyse}

In Anlehnung an die gegenständliche Gliederung der Kultur von Posner (1991) lassen sich kulturelle Erscheinungen über Texte, Zeichen und Artefakte einordnen, also in erster Linie in ihrer sichtbaren materialen Dimension beschreiben. So liegt es nahe, die kulturbasierte MVC-Analyse mit dem Bereich der Alltagskultur (Essen, Mode, Musik, Wohnen/Interieur) zu beginnen. Dabei können v. a. Artefakte und alltägliche Gegenstände, die in einem MVC vorkommen, thematisiert sowie ihre Bedeutung für die Interpretationen des Videos geklärt werden. Auf dieser Analyseebene ist allgemeines soziokulturelles Orientierungswissen gefragt. Für den MVC von Gazmanov Vpered, Rossija bedeutet das z. B., dass die LernerInnen bei der visuellen Ebene Orte des kollektiven Gedächtnisses (die Mutter-Heimat-Statue in Volgograd, den Roten Platz und die Christi-ErlöserKathedrale in Moskau) erkennen. Bezeichnenderweise werden die Erfolge der Vergangenheit im Videoclip von Gazmanov in einer einfachen Bildsprache immer wieder in Verbindung zum aktuellen Russland gebracht - die olympischen Spiele in Moskau 1980 und jene in Soči 2014, die sowjetische und die heutige Raumfahrt, die Helden der Sowjetunion und die Soldaten oder die erfolgreichen Sportler der Gegenwart. Als Superheld wird dabei Vladimir Putin gefeiert, der als ultimativer, von der Masse bejubelter Star dargestellt wird. Auf der auditiven Ebene fällt auf, dass bereits die erste Strophe, die sich auf einen der bekanntesten und mehrmals verfilmten Romane des Sozialistischen Realismus Kak zakaljalas' stal'von N. A. Ostrovksij (1932-1934) bezieht, den Grundton der Inszenierung angibt. Und zwar: die Glorifizierung der Sowjetunion als Imperium. Historische Kontinuität wird bereits in der Klammer zum eigentlichen Video hergestellt, indem die Melodie des patriotischen Liedes Slav'sja von Michail Glinka (aus der Oper Ivan Susanin, Uraufführung 1836) als Prolog und Epilog eingespielt wird. Trotz der Akzentuierung der für die zaristische und die heutige Zeit identitäts-

Anka Bergmann, Olga Caspers \& Wolfgang Stadler (Hg.)

Didaktik der slawischen Sprachen - Beiträge zum 1. Arbeitskreis in Berlin (12.-14.9.2016)

(C) 2018 innsbruck university press, ISBN 978-3-903187-11-5, DOI 10.15203/3187-11-5 
stiftenden Orthodoxie wird im MVC von Gazmanov Russland als multireligiöser Staat inszeniert.

Für das Verständnis des Videos Patriot von Nesčastnyj slučaj ist die Wiedererkennung der Figur von Nikita Michalkov durch die LernerInnen besonders wichtig. Der prominente Regisseur, der sich gern in eigenen Filmen als Vaterfigur der Nation inszeniert und die untrennbare Verbindung von russischer Kultur, russischer Orthodoxie und russischem Staat predigt (Schmid 2015: 185), gehört zu den Schlüsselfiguren im heutigen Russland, die die kulturelle, politische und ideologische Szenerie des Landes bedeutend prägen.

Ausgehend von Persönlichkeiten, historischen Ereignissen und Artefakten können sodann empirisch wahrnehmbare kulturelle Akte, d. h. Soziofakte (Verhaltensmuster, kommunikatives Handeln) beschrieben werden. Dazu gehören in den MVCs der offiziellen Kultur z. B. Hierarchien in der politischen Kommunikation (Putin als Symbolfigur und ,höchster Wert', der erste Mann an der Spitze als Vaterfigur, als glamouröser Star etc.) oder auch patriarchale Muster, die sich in der Alltagskommunikation und einem genderspezifischen Verhalten widerspiegeln. In den satirischen Inszenierungen ist dieses kommunikative Handeln - da überspitzt dargestellt - deutlich erkennbar (wie das Dating-Verhalten im MVC Ekksponat oder exzessives Feiern im MVC Party Like A Russian). In den Videoclips können ferner die nonverbalen Muster der Kommunikation beobachtet werden - Begrüßungs- und Berührungsrituale, persönliche Distanz oder etwa das Phänomen des (Nicht-)Lächelns in der russischen Kultur, welches Robbie Williams in seinem Videoclip besonders gut aus der Fremdperspektive flankiert. An dieser Stelle bilden Hofstedes Parameter (Hofstede 2011 u. a.), die in Form binärer Opposition formuliert sind (Kollektivismus vs. Individualismus, Maskulinität vs. Feminität, lang- vs. kurzfristige Planung u. a.), eine gute Grundlage, um die Leitfragen im Bereich der Verhaltenscodes zu diskutieren und kritisch zu hinterfragen (vgl. Caspers 2016).

Schließlich ist ein System von Werten und Konventionen zu beachten, die eine mentale Kultur ausmachen (Posner 2003: 53). Um diese nicht direkt wahrnehmbaren Größen im Unterricht zu exemplifizieren, bietet es sich an, mit Begriffen der russischen Konzeptologie (vgl. Karasik \& Sternin 2005-2011,

Anka Bergmann, Olga Caspers \& Wolfgang Stadler (Hg.)

Didaktik der slawischen Sprachen - Beiträge zum 1. Arbeitskreis in Berlin (12.-14.9.2016)

(C) 2018 innsbruck university press, ISBN 978-3-903187-11-5, DOI 10.15203/3187-11-5 
Zaliznjak, Levontina \& Šmelev 2012) ${ }^{10}$ zu arbeiten. Als ergiebig erweist sich bspw. die Thematisierung der „russischen Weite“ (prostor, vgl. Šmelev 2012), die als ein bekanntes Auto- und Heterostereotyp beinahe in allen MVCs über Russland vorkommt und in der autostereotypen Perspektive nicht nur die territoriale Vorstellung von der Größe des Landes, sondern auch vom russischen Charakter und der ,russischen Seele‘ impliziert (vgl. Kuße 2012: 61-63).

Damit wird deutlich, dass bereits die Berücksichtigung formaler Kriterien bei einer MVC-Analyse Sensibilisierungsprozesse für das kulturspezifische ,Andere' in Gang setzen kann. Intensiviert werden diese Prozesse, wenn man auch funktionale und evaluative Parameter heranzieht. Als übergeordnetes analytisches Instrumentarium greift hier die funktionale Gliederung von Kultur, die eine Ausdifferenzierung in einen privaten, offiziellen und öffentlichen Bereich vorsieht (Kuße 2012: 31-35). Denn soziale, mentale und kulturelle Gegenstände können je nach institutionellem Bereich unterschiedliche Funktionen erfüllen und unterschiedliche Grade an Kulturalität im Sinne kultureller Markierungen aufweisen (ebd.: 32). Die Popmusikvideos changieren dabei auf bemerkenswerte Weise zwischen den funktionalen Sphären der Kultur.

Im Fernsehen werden patriotische Lieder auf Festkonzerten in Form einer ritualisierten medienkulturellen Praxis dargeboten, sie gelten als Schlager und werden - wie viele andere populäre Lieder - außerhalb des TV-Konsums sowohl auf öffentlichen Veranstaltungen, z. B. in Schulen und Karaoke-Bars, als auch privat, unter Freunden oder allein gesungen. Sie verbinden die Sphäre des Festtags mit jener des Alltags und tradieren außerdem Melodien, die im kollektiven Bewusstsein verankert sind und Bezüge zur Volkskultur aufwerfen. Ein hoher Grad an Kulturalität lässt sich im Genre der russischen Estrada am Beispiel der inflationär gebrauchten Kriegssymbolik und übertriebenen militanten Inszenierungen erläutern (insb. in MVCs von Gazmanov). Auch Begriffe wie „Rodina“ und "Otečestvo" weisen Besonderheiten im semantischen Gehalt sowie in der pragmatischen Verwendung auf und vermitteln wichtige landeskundliche Infor-

10 Die in der kognitivistischen Linguistik beschriebene Konzeptosphäre liefert eine Reihe von Beispielen für kulturelle Konstanten, die relativ gut exploriert sind, aber bisher weder theoretische noch praktische Anwendung in der deutschsprachigen Fachdidaktik des Russischen finden.

Anka Bergmann, Olga Caspers \& Wolfgang Stadler (Hg.)

Didaktik der slawischen Sprachen - Beiträge zum 1. Arbeitskreis in Berlin (12.-14.9.2016)

(C) 2018 innsbruck university press, ISBN 978-3-903187-11-5, DOI 10.15203/3187-11-5 
mationen. Als Teil der offiziellen Kultur akzentuieren die patriotischen MVCs ein positives Selbstbild von Russland, sie adressieren einen sense of community und sollen gemeinschaftsstiftend wirken.

Trotz dieser kulturspezifischen Markierung stellen Estrada-Lieder eine transkulturelle Erscheinung dar und bieten eine geeignete Plattform, von welcher aus das In-Beziehung-Setzen des Eigenen mit dem Fremden erfolgen kann. Nimmt man den Hurra-Patriotismus näher in den Blick, so tritt in unseren Beispielen Glamour als gemeinsame ästhetische Klammer auf (s. Abschnitt 3). Gerade bei der Analyse dieser Ebene lässt sich der Blick vom Vertrauten auf kulturelle Bruchstellen lenken. Bsps. wird unter Rekurs auf Hollywood-Ästhetik in russischen patriotischen MVCs und damit einhergehend im aktuellen politischen Diskurs Russlands Aggression (Kriegsinszenierungen, militante Symbolik und konkrete militärische Auseinandersetzungen) als Prestige dargestellt. An MVCs von Gazmanov lässt sich also zeigen, wie der ästhetische Modus des Glamours für politische Zwecke instrumentalisiert wird.

Anders verhält es sich in den MVCs der Gruppe Leningrad. Hier wird die Scheinwelt des Glamours durch Imitation und Fälschungen der Alltagsgegenstände (insb. Schuhe der Marke Louboutin) ironisch bloßgestellt. Der MVC liefert darüber hinaus zahlreiche Beispiele für Kulturtransfer, welcher im universitären Unterricht vor dem Hintergrund der Transkulturalitätsthese thematisiert werden kann (als Beispiel ist hier die Wunschwand der Protagonistin zu nennen, auf der Bilder von Victoria Beckham, Prinz William und seiner Frau Kate, Britney Spears und Julia Roberts als Vorbilder zu sehen sind). In Korrelation damit steht der Videoclip von Robbie Williams, der einen ,typischen' Russen als einen reichen und exzentrischen Oligarchen karikiert und auf vielfältige Weise russische Heterostereotype bedient. Deutlich zum Tragen kommt dabei die evaluative Komponente. Bei der MVC-Analyse bietet es sich daher an, insb. darauf zu achten, wie VertreterInnen der eigenen und zielsprachigen Kulturen dargestellt werden, wie Freund- und Feindbilder konstruiert und dekonstruiert werden, wie die Erzeugung von Empathie stattfindet und schließlich auf welche Art und Weise Humor, Angst oder Toleranz vermittelt werden. Durch kritische Reflexion können diese konstruierten Bilder überprüft und auf der Makro- und Mikroebene analysiert werden.

Anka Bergmann, Olga Caspers \& Wolfgang Stadler (Hg.)

Didaktik der slawischen Sprachen - Beiträge zum 1. Arbeitskreis in Berlin (12.-14.9.2016)

(C) 2018 innsbruck university press, ISBN 978-3-903187-11-5, DOI 10.15203/3187-11-5 


\subsection{Phasen der MVC-Analyse}

Die Thematisierung der oben beschriebenen Dimensionen lässt sich wie folgt methodisch gestalten:

Im universitären kulturvermittelnden Unterricht ist es sinnvoll, sich in der Input-Phase mit theoretischen Grundlagen auseinanderzusetzen, um so eine Analysebasis zu schaffen. Welcher der oben vorgestellten Ansätze als Schwerpunkt gewählt wird, hängt von der Auswahl der MVCs und den anvisierten Unterrichtszielen ab. Grundsätzlich ist festzuhalten, dass bei der Arbeit mit Musikvideos der Bezug auf mehrere Theorien am ehesten der Mehrdimensionalität und Komplexität des Gegenstandes gerecht wird. Dabei kann bspw. die in der literaturwissenschaftlichen Textanalyse entwickelte Methode des close reading durch close viewing und close listening ergänzt werden.

Für die pre-viewing activities wird oft vorgeschlagen, über die Bedeutung der Songtitel oder über Möglichkeiten der visuellen Umsetzung eines Liedes zu diskutieren (Volkmann 2007: 289). Man kann die LernerInnen in Gruppen einteilen und sie anhand von Bildern und Titeln Hypothesen über den möglichen Inhalt aufstellen lassen. Die aufgestellten Hypothesen sollen im Kontext vorhandener Erfahrungen und Kenntnisse im interkulturellen Bereich diskutiert werden. In dieser Arbeitsphase ist es wichtig, die affektive Ebene anzusprechen, um auf die bereits vorhandenen eigenen Erfahrungen und Wertvorstellungen der LernerInnen zu rekurrieren. Die MVC-Analyse kann man mit Bildern einleiten, indem in einer sound-off-Präsentation nur die visuelle Ebene gezeigt wird. Will man die Textebene in die Analyse einbeziehen, so gilt die Aufmerksamkeit in der pre-viewing-Phase der lexikalischen Vorentlastung.

Als while-viewing activities erscheinen text- oder bildbezogene Aufgaben sinnvoll. Bei der Videovorführung soll die Aufmerksamkeit der LernerInnen auf relevante Ereignisse anhand von vorab formulierten Fragen gelenkt werden. Dazu bieten sich z. B. freeze-frame-Aktivitäten an (das Anhalten des Videos an einer Stelle und Fragen zur Analyse, Fragen zum möglichen weiteren Verlauf). In dieser Arbeitsphase kann ebenfalls die Klärung der ,dunklen Stellen' beginnen. Was wurde nicht verstanden? In der Kultur: Welche (verdeckten) kulturellen Markierungen, Voraussetzungen oder auch impliziten Botschaften, die mitkonsumiert werden,

Anka Bergmann, Olga Caspers \& Wolfgang Stadler (Hg.)

Didaktik der slawischen Sprachen - Beiträge zum 1. Arbeitskreis in Berlin (12.-14.9.2016)

(C) 2018 innsbruck university press, ISBN 978-3-903187-11-5, DOI 10.15203/3187-11-5 
nimmt man durch die verlangsamte Wahrnehmung wahr? Welche ,fremdspezifischen' Eigenheiten und Unterschiede zum Eigenkulturellen werden sichtbar?

Für die post-viewing activities sind textproduktive Aufgaben, dialogische, aber auch musikalische und visuelle Aufgaben (z. B. Erstellung eines storyboards) geeignet. In dieser Phase ist es wichtig, dass zu Beginn aufgestellte Hypothesen verifiziert werden. Ferner soll man sich vergewissern, dass das Detailverstehen gesichert ist. Erst im nächsten Schritt folgen die Arbeitsformen, die für das interkulturelle Lernen zentral sind, und zwar die Diskussion der Ereignisse aus verschiedenen Erzählperspektiven. Durch Perspektivenwechsel kann man bei dieser Art von Aufgaben eine Veränderung der Einstellung zur jeweiligen Problematik erzielen. Im Anschluss bietet es sich an, LernerInnen in Gruppen arbeiten und eine gemeinsame Analyse eines MVCs aus dem Korpus anhand eines gezielten Fragenkatalogs durchführen zu lassen, die später im Plenum diskutiert wird. Die Fragen sollen so formuliert sein, dass die Fähigkeit zur Selbstbeobachtung und Selbstreflexion trainiert wird. Hier wird empfohlen, die Fragen im ersten Block lernerorientiert zu formulieren, sodass sie die subjektive Erfahrung von Studierenden aktivieren und eine kreative Hypothesenbildung stimulieren. Der nächste Block im Fragenkatalog kann eher prozessorientiert ausgerichtet sein und soll zur kognitiven Bewusstseinserweiterung durch das Einordnen neuer Informationen in bereits vorhandene Wissensstrukturen beitragen sowie zum Vergleich mit der eigenen Kultur anleiten. Der letzte Block der Fragen ist dagegen handlungsorientiert zu gestalten, um LernerInnen dazu zu bewegen, eigene Strategien im Umgang mit den MVC zu entwickeln.

Nach einer ausführlichen Beschäftigung mit interkulturellen Inhalten folgt die Output-Phase, die sich durch die individuelle Arbeit am MVC kennzeichnet und u. a. die Vorbereitung und Durchführung einer Präsentation zum ausgewählten MVC beinhalten kann. Die LernerInnen führen eine MVC-Analyse selbstständig durch, wobei sich der Vergleich eines Videos aus dem Korpus mit einem thematisch verwandten MVC aus der eigenen Kultur als ergiebig erweist. Bei der Evaluation soll insb. darauf geachtet werden, dass die Aufgaben zur Wiedergabe von soziokulturellem Wissen, Aufgaben zur Feststellung von Methodenund Theorienkenntnis, Aufgaben zum reflektierten Vergleich kultureller Phänomene (z. B. nonverbale Kommunikation) etc. entsprechend bearbeitet werden.

Anka Bergmann, Olga Caspers \& Wolfgang Stadler (Hg.)

Didaktik der slawischen Sprachen - Beiträge zum 1. Arbeitskreis in Berlin (12.-14.9.2016)

(C) 2018 innsbruck university press, ISBN 978-3-903187-11-5, DOI 10.15203/3187-11-5 
Bei der Arbeit mit MVCs, die im Internet zugänglich sind und von den UserInnen kommentiert werden, können Lernende selbstständig Recherchen zu bestimmten Aspekten der Populärkultur machen. Dabei kann deutlich werden, „wie unterschiedlich die Angebote der medialen Popkultur - je nach Alter, sozialer Schicht und nationaler oder ethnischer Herkunft - die Menschen im elektronischen Zeitalter in ihren Bann schlagen und zur jeweiligen Identitätskonstruktion beitragen“ (Volkmann 2007: 289). Zudem zeigt sich an diesen Beispielen und deren theoretischer Einbettung, wie Kultur als dynamisches Phänomen funktioniert.

\section{Schlussbetrachtung}

Die Auseinandersetzung mit populären Musikvideos verschafft Orientierung in der russischen Massenkultur und ermöglicht ein besseres Verstehen gesellschaftspolitischer Prozesse. Somit wird ein Beitrag zur Erweiterung des medialen Spektrums im Bereich Russistik/Russisch als Fremdsprache geleistet. Über die Vermittlung von Fremdsprache, zielkulturellem Wissen und die Herausbildung interkultureller Kompetenzen hinaus trägt der Fremdsprachenunterricht - so unser Fazit - im Wesentlichen zum gezielten und reflektierten Umgang mit Medien bei. Die theoriegeleitete Herangehensweise an MVCs setzt sich zum Ziel, die Interpretations- und Analysekompetenz der LernerInnen auszubauen und ihre Strategienkompetenzen zu stärken. Zu diesem Zweck muss es sich auch die russistische Kulturdidaktik zur Aufgabe machen, den Studierenden sowie den LehrerInnen in Aus- und Fortbildung professionelles Wissen in Hinblick auf aktuelle und ansprechende Untersuchungsgegenstände, Arbeitstechniken und Methoden sowie die dazugehörenden Theorien zur Verfügung zu stellen. Angesichts der zunehmenden Institutionalisierung kulturwissenschaftlicher Veranstaltungen, die in der Slawistik zu beobachten ist, wäre es wünschenswert, dass Kulturwissenschaft stärker als bisher hochschuldidaktische Probleme thematisiert, aber auch die Fachdidaktik die Erkenntnisse dieser Bezugsdisziplin in gegenläufiger Perspektive nutzt. 


\section{Literaturverzeichnis}

Bachmann-Medick, D. (1996). Einleitung. In Ders. (Hrsg.), Kultur als Text. Die anthropologische Wende in der Literaturwissenschaft (7-64). Frankfurt a. M.: Fischer.

Barker, A. M. (1999). The Culture Factory: Theorizing the Popular in the Old and New Russia. In A. M. Barker (Hrsg.), Consuming Russia. Popular Culture, Sex, and Society (12-45). Durham, London: Duke University Press.

Blell, G. (2002). Musikvideoclips im Englischunterricht: ,medialer Himmel' oder ,mediale Hölle‘? In H. Decke-Cornill \& M. Reichert-Wallrabenstein (Hrsg.), Fremdsprachenunterricht in medialen Lernumgebungen (195-208). Frankfurt a. M.: Peter Lang.

Blell, G. (2005). Fremdsprachenlernen zwischen Medienverwahrlosung und Medienkompetenz: Beiträge zu einer kritisch-reflektierenden Mediendidaktik. Frankfurt a. M.: Peter Lang.

Caspers, O. (2016). The Cultural Dialogue in Vera Storozheva's Cinematographic Works: Teaching Russian Values in the Foreign Language Class with the Example of Contemporary Russian Films. In O. Laguta \& A. Savchenko (Hrsg.), Slavica Formosana I: Humanitarian Values in Slavic Languages and Cultures. Collection of Research Papers of the VI International Conference "Modern Problems of Slavic Philology: Humanitarian Values in Slavic Languages and Cultures" (405-430). Taipei: National Chengchi University.

Doschek, J. \& Simonek, S. (2015). Slawische Popkultur (Band 2). Wien: Polnische Akademie der Wissenschaften.

Dubin, B. (2006). Simulierte Macht und zeremonielle Politik. Osteuropa, 3, 19-32.

Dubin, B. (2010). Klassika, posle i rjadom: Sociologičeskie očerki o literature i kul'ture. Moskau: Novoe literaturnoe obozrenie.

Eisenmann, M. (2010). „in your head they are fighting“ - Anregungen zur Arbeit mit Muskivideoclips am Beispiel des Songs „Zombie“. In G. Blell \& R. Kupetz (Hrsg.), Der Einsatz von Musik und die Entwicklung von audio literacy im Fremdsprachenunterricht (251-262). Frankfurt a. M.: Peter Lang.

Frazer, P. (2005). Teaching Music Video. London: British Film Industrie.

Goscilo, H. \& Strukov, V. (2010). Celebrity and Glamour in Contemporary Russia. Abingdon: Routledge.

Gussarowa, X. (2008). Norm und Abweichung: Glamour in der Mode. Kultura. Russland-Kulturanalysen, 6, 4-9.

Anka Bergmann, Olga Caspers \& Wolfgang Stadler (Hg.)

Didaktik der slawischen Sprachen - Beiträge zum 1. Arbeitskreis in Berlin (12.-14.9.2016)

(C) 2018 innsbruck university press, ISBN 978-3-903187-11-5, DOI 10.15203/3187-11-5 
Hallet, W. \& Nünning, A. (2007). Neue Ansätze und Konzepte der Literatur- und Kulturdidaktik (Band 1). Trier: WVT.

Hofstede, G. (2011). Dimensionalizing Cultures: The Hofstede Model in Context. Online Readings in Psychology and Culture. Verfügbar unter: https://tinyurl.com/y7qqnnzs [02.12.2017].

$\mathrm{Hu}$, A. (2007). Kulturwissenschaftliche Ansätze in der Fremdsprachendidaktik. In W. Hallet \& A. Nünning (Hrsg.), Neue Ansätze und Konzepte der Literatur-und Kulturdidaktik (Band 1) (13-30). Trier: WVT.

Kanzler, K. \& Scharlaj, M. (2017). Between Glamorous Patriotism and Reality-TV Aesthetics: Political Communication, Popular Culture, and the Invective Turn in Trump's United States and Putin's Russia. Zeitschrift für Slawistik, 2017/2, 316-338.

Karasik, V. I. \& Sternin, I. A. (2005-2011). Antologija konceptov (Tom 1-8). Volgograd: o. V.

Kuße, H. (2012). Kulturwissenschaftliche Linguistik. Eine Einführung. Göttingen: Vandenhoeck \& Ruprecht.

Leitzke-Ungerer, E. (Hrsg.) (2009). Film im Fremdsprachenunterricht. Literarische Stoffe, interkulturelle Ziele, mediale Wirkung. Stuttgart: Ibidem.

Lotman, Ju. M. (1990). Über die Seimosphäre. Zeitschrift für Semiotik, 12, (4), 287-305.

Mehlhorn, G. (2014). Interkulturelle Kompetenz entwickeln. In A. Bergmann (Hrsg.), Fachdidaktik Russisch. Eine Einführung (214-227). Tübingen: Narr.

Menzel, B. (2013). Russischer Glamour und die Ära Putin. Russland-Analysen, 258, 31.05.2013, 6-11.

Themenheft Osteuropa (2007). Der Osten im Westen. Importe der Populärkultur 2007/5.

Posner, R. (1991). Kultur als Zeichensystem. Zur semiotischen Explikation kulturwissenschaftlicher Grundbegriffe. In A. Assmann \& D. Harth (Hrsg.), Kultur als Lebenswelt und Monument (37-74). Frankfurt a. M.: Fischer.

Posner, R. (2003). Kultursemiotik. In A. Nünning \& V. Nünning (Hrsg.), Konzepte der Kulturwissenschaften (39-72). Stuttgart \& Weimar: Metzler.

Reimann, D. (2014). Musikvideoklips und fremdsprachliches Hör-/Sehverstehen. Authentische Sprache, Texte und Bilder im Französisch-, Spanisch- und Italienischunterricht. In Ders. (Hrsg.), Transkulturelle kommunikative Kompetenz in den romanischen Sprachen (365-385). Stuttgart: Ibidem.

Rozenchol'm, A. \& Savkina, I. (Hrsg). (2015). Topografii populjarnoj kul'tury. Moskva: Novoe literaturnoe obozrenie.

Rudova, L. (2008). Russland - in Glamour vereint. Kultura. Russland-Kulturanalysen, 6, 2-3.

Anka Bergmann, Olga Caspers \& Wolfgang Stadler (Hg.)

Didaktik der slawischen Sprachen - Beiträge zum 1. Arbeitskreis in Berlin (12.-14.9.2016)

(C) 2018 innsbruck university press, ISBN 978-3-903187-11-5, DOI 10.15203/3187-11-5 
Scharlaj, M. (2017). Krieg, Kommerz und Kremlkonzerte. Geschichtsinszenierungen im heutigen Russland. In Th. Kühn \& R. Troschitz (Hrsg.), Populärkultur. Perspektiven und Analysen (157-179). Bielefeld: transcript.

Schmid, U. (2016). Technologien der Seele. Vom Verfertigen der Wahrheit in der russischen Gegenwartskultur. Berlin: Suhrkamp.

Šmelev, A. D. (2012). Prostranstvennaja sostavljajuščaja russkoj kul'tury. In H. Kuße \& M. Scharlaj (Hrsg.), Prostransto i dom v russkom jazyke i v russkoj kul'ture. Raum und Haus in der russischen Sprache und Kultur (11-26). München et al.: Otto Sagner.

Sommer, R. (2007). Vom ,Survey zum ,Sample': Kulturdidaktische Modelle zwischen Landeskunde, interkulturellem Lernen und Kulturwissenschaft. In W. Hallet \& A. Nünning (Hrsg.), Neue Ansätze und Konzepte der Literatur- und Kulturdidaktik (Band 1) (183-195). Trier: WVT.

Thaler, E. (1999). Musikvideoklips im Englischunterricht. Phänomenologie, Legitimität, Didaktik und Methodik eines neuen Mediums. München: Langenscheidt-Longman.

Thaler, E. (2012). Musikvideoklips - Kunst, Kitsch, Kommerz, Kommunikation. In G. Blell \& R. Kupetz (Hrsg.), Der Einsatz von Musik und die Entwicklung von audio literacy im Fremdsprachenunterricht (241-250). Frankfurt a. M.: Peter Lang.

Trepper, H. (2002). Einleitung. Von der „Massenkultur“ zur „Popularkultur“. In I. Bock, W. Schlott \& H. Trepper (Hrsg.), Kommerz, Kunst, Unterhaltung. Die neue Popularkultur in Zentral- und Osteuropa (7-22). Bremen: Edition Temmen.

Volkmann, L. (2007). Popular Culture im Fremdsprachenunterricht: Musicvideoclips, Popsong, Werbung. In W. Hallet \& A. Nünning (Hrsg.), Neue Ansätze und Konzepte der Literatur-und Kulturdidaktik (Band 1) (277-291). Trier: WVT.

Welsch W. (2010). Was ist eigentlich Transkulturalität? In L. Darowska, T. Lüttenberg \& C. Machold (Hrsg.), Hochschule als transkultureller Raum? Kultur, Bildung und Differenz in der Universität (39-66). Bielefeld: transcript.

Willwer, J. (2009). Musikclips zu Bénabar im Französischunterricht. In E. Leitzke-Ungerer (Hrsg.), Film im Fremdsprachenunterricht (399-417). Stuttgart: Ibidem.

Zaliznjak, A., Levontina I. \& Šmelev, A. (2012). Konstanty i peremennye russkoj jazykovoj kartiny mira. Moskau: o. V.

Zvereva, V. (2008). Glamur v sovremennoj rossijskoj kul'ture. In A. M. Nikulin (Hrsg.), Puti Rossii: kul'tura - obšcestvo - čelovek. Materialy Meždunarodnogo simpoziuma (25-26 janvarja 2008 goda) (128-138). Moskau: Logos.

Anka Bergmann, Olga Caspers \& Wolfgang Stadler (Hg.)

Didaktik der slawischen Sprachen - Beiträge zum 1. Arbeitskreis in Berlin (12.-14.9.2016)

(C) 2018 innsbruck university press, ISBN 978-3-903187-11-5, DOI 10.15203/3187-11-5 
